\section{Troubles cognitifs et infection par le VIH-1}

\section{La protéine Tat altère la neurosécrétion}

Petra Tryoen-Tóth ${ }^{1}$, Bruno Beaumelle ${ }^{2}$, Marie-France Bader ${ }^{1}$, Nicolas Vitale ${ }^{1}$
${ }^{1}$ Institut des neurosciences cellulaires et intégratives ; CNRS UPR-3212, 5, rue Blaise Pascal, 67084 Strasbourg; Université de Strasbourg, Strasbourg, France; ${ }^{2} \mathrm{CPBS}$ - Centre d'étude d'agents pathogènes et biotechnologies pour la santé ; UMR 5236 CNRS ; Université Montpellier 1 et $2 ; 1919$, route de Mende, 34293 Montpellier Cedex 5, France. vitalen@inci-cnrs.unistra.fr petra.tryoen@inci-cnrs.unistra.fr
> Malgré l'identification de l'agent causal du syndrome d'immunodéficience acquise (Sida), un rétrovirus baptisé VIH-1 (pour virus de l'immunodéficience humaine 1) et la mise au point d'un traitement efficace à base de molécules antivirales, l'éradication complète du virus semble toujours hors de portée [1]. Près d'un tiers des patients atteints de Sida et sous trithérapie présentent des dysfonctionnements moteurs et cognitifs, ainsi que des modifications du comportement, et un sur dix développe une démence liée au VIH-l dans les dernières phases de la maladie [2]. Bien que certains signes de mort neuronale aient été mis en évidence chez ces patients, I'ampleur des détériorations cognitives suggère l'existence d'un ou plusieurs autres mécanismes conduisant à une altération des communications neuronales. Dans ce contexte, nous décrivons dans cette Nouvelle les interactions moléculaires par lesquelles la protéine Tat du VIH-l est capable de bloquer la transmission neuroendocrine et neuronale.

\section{Fonction de Tat dans l'infection par le VIH-1}

Le VIH-l est un lentivirus de la famille des rétrovirus dont l'information génétique, codée sous forme d'ARN, est rétrotranscrite en ADN double brin avant d'être intégrée dans le génome de la cellule hôte. Le génome du VIH est composé de neuf gènes, dont trois gènes de structure communs à tous les rétrovirus ( $g a g$, pol et env) et six gènes codant pour des protéines régulatrices (nef, rev, tat, vif, vpr et vpu). Parmi ces gènes, tat code pour une protéine de 86 à 103 acides aminés selon les isolats viraux ; en potentialisant l'efficacité de la transcription virale, Tat permet une production virale puissante qui est déterminante pour vaincre la réponse immunitaire de l'hôte.

Tat semble également jouer un rôle direct dans différentes pathologies associées au Sida. Bien qu'elle soit sécrétée par les cellules infectées directement à travers la membrane plasmique, et donc selon un mode non conventionnel, Tat est présente à des concentrations nanomolaires dans le sang des patients infectés par le VIH [3]. Elle possède une courte séquence peptidique basique lui permettant de pénétrer dans les cellules [4], et il est généralement admis que la protéine Tat circulante puisse agir comme une toxine en pénétrant des cellules non infectées pour y modifier leur physiologie. Ces effets incluent notamment une augmentation de l'expression de certains corécepteurs du VIH, et la production de cytokines par les lymphocytes, monocytes et macrophages conduisant à une immunosuppression. Cette internalisation de Tat semble faire intervenir différents mécanismes d'endocytose en fonction du type cellulaire. Tat peut ensuite, comme certaines toxines bactériennes, traverser la membrane endosomale pour gagner le cytosol des cellules cibles. Tat entre ainsi dans les neurones et les cellules neuroendocrines $[5,6]$.
Tat inhibe la neurosécrétion en perturbant le processus d'exocytose Les cellules neurosécrétrices libèrent les neurotransmetteurs et les hormones qu'elles stockent dans des vésicules/ granules membranaires par exocytose en réponse à la stimulation de récepteurs présents à la surface cellulaire. Nous avons récemment montré qu'une exposition de cellules neuroendocrines de la médullosurrénale en culture (les cellules chromaffines) à la protéine Tat induit une forte altération de leur activité d'exocytose des catécholamines [6]. De même, en utilisant un marqueur de vésicules synaptiques, la sonde FMl-43, nous avons observé que Tat bloque le cycle d'exocytose/endocytose des vésicules synaptiques et la libération de transmetteurs par les neurones corticaux en culture [6]. Cette capacité de Tat à bloquer la neurosécrétion dépend de sa capacité à être internalisée et à lier avec une très forte affinité le phospholipide phosphatidylinositol 4,5-bisphophate [Ptdlns $(4,5) \mathrm{P}_{2}$ ou PIP2] présent sur la face interne de la membrane plasmique [6, 7]. Or, ce lipide joue un rôle crucial dans diverses étapes du processus d'exocytose, et notamment dans le recrutement des vésicules et la formation des sites d'exocytose au niveau de la membrane plasmique [8]. De fait, l'effet inhibiteur de Tat sur la neurosécrétion peut être levé en surexprimant dans les cellules une kinase capable de synthétiser et d'augmenter le taux de Ptdlns $(4,5) P_{2}$. Inversement, I'accumulation de Tat à la membrane plasmique et son effet inhibiteur sur l'exocytose sont 


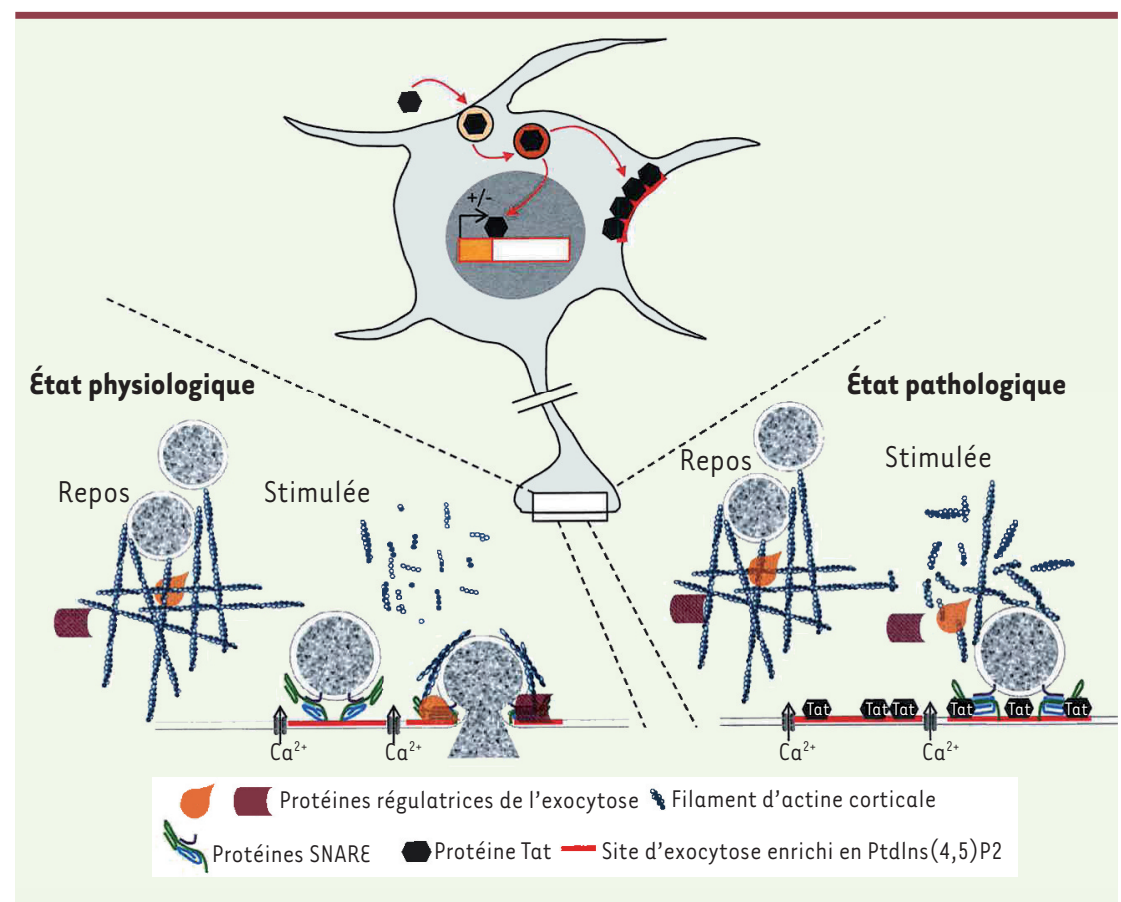

Figure 1. Modèle du mécanisme permettant à Tat d'inhiber la neurosécrétion. Dans les conditions physiologiques normales, la stimulation des cellules neurosécrétrices entraîne une élévation du niveau intracellulaire de calcium qui conduit à une modification de l'état de polymérisation des filaments d'actine corticale. Ceci permet le recrutement des vésicules de sécrétion à la membrane plasmique où se forment les sites d'exocytose impliquant le complexe multiprotéique SNARE et des protéines régulatrices. Ces deux processus nécessitent des interactions avec le phospholipide Ptdlns $(4,5) \mathrm{P}_{2}$. Dans des conditions pathologiques, Tat peut pénétrer dans les cellules neurosécrétrices par endocytose et se retrouver dans le noyau pour y affecter la transcription de certains gènes. En se liant au Ptdlns $(4,5) P_{2}$ de la face interne de la membrane plasmique, Tat altère la dépolymérisation des filaments d'actine corticale et la formation des sites d'exocytose fonctionnels.

empêchés par une mutation de Tat qui abolit sa liaison au Ptdlns $(4,5) P_{2}[6]$. Diverses approches biophysiques ont permis d'établir que la fixation de Tat sur le Ptdlns $(4,5) P_{2}$ s'accompagne de l'ancrage de la chaîne latérale du résidu tryptophane 11 dans la membrane lipidique [7]. Cela explique la très forte affinité de Tat pour ce phospho-inositide. Élément structural et régulateur des plates-formes d'arrimage des vésicules de sécrétion au niveau de la membrane plasmique, le $\operatorname{Ptdlns}(4,5) \mathrm{P}_{2}$ joue un rôle central dans les mécanismes de l'exocytose. II contrôle, entre autres, l'activité de protéines impliquées dans l'étape d'arrimage précédant la fusion des membranes vésiculaire et plasmique. II module également l'organisation du cytosquelette d'actine dont l'intervention dans les étapes successives nécessite également son interaction avec le $\operatorname{Ptdlns}(4,5) \mathrm{P}_{2}[10]$. Par ailleurs, l'accumulation de Tat dans les cellules neurosécrétrices suggère l'existence possible d'un mécanisme de rétention spécifique qu'il sera pertinent d'identifier. En conclusion, l'altération de la neurosécrétion par Tat peut directement conduire à une détérioration des transmissions synaptiques, voire à la mort neuronale à plus long terme, et ainsi contribuer aux perturbations neuronales observées chez les patients infectés par le VIH. Ces résultats laissent également entrevoir la possibilité de traiter ces symptômes neurologiques en neutralisant la protéine Tat. $\diamond$

HIV-associated cognitive disorders: Tat perturbs neurosecretion

\section{RÉFÉRENCES}

1. Schwartz C, Le Douce V, Cherrier T, et al. Un virus tapi dans l'ombre: les bases moléculaires de la latence du VIH-1 - Partie I : la physiologie de la latence du VIH-1. Med Sci (Paris) $2010 ; 26: 159-63$

2. Ghafouri M, Amini S, Khalili K, Sawaya BE. HIV-1 associated dementia : symptoms and causes. Retrovirology $2006 ; 3: 28$.

3. Xiao H, Neuveut C, Tiffany HL, et al. Selective CXCR4 antagonism by Tat: implications for in vivo expansion of coreceptor use by HIV-1. Proc Natl Acad Sci USA $2000 ; 97: 11466-71$.

4. Frankel AD, Pabo CO. Cellular uptake of the tat protein from human immunodeficiency virus. Cell 1988 ; 55 : 1189-93.

5. Liu Y, Jones M, Hingtgen CM, et al. Uptake of HIV-1 tat protein mediated by low-density lipoprotein receptor-related protein disrupts the neuronal metabolic balance of the receptor ligands. Nat Med $2000 ; 6: 1380-7$

6. Tryoen-Tóth P, Chasserot-Golaz S, Tu A, et al. HIV-1 Tat protein inhibits neurosecretion by binding to phosphatidylinositol 4,5-bisphosphate. J Cell Sci 2013 ; $126: 454-63$

7. Rayne F, Debaisieux S, Yezid H, et al. Phosphatidylinositol-(4,5)-bisphosphate enables efficient secretion of HIV-1 Tat by infected T-cells. EMBO J 2010 ; 29 : 1348-62.

8. Chasserot-Golaz S, Coorssen JR, Meunier FA, Vitale N. Lipid dynamics in exocytosis. Cell Mol Neurobiol 2010 ; 30 : 1335-42.

9. Vitale N, Bader MF, Beaumelle B, Tryoen-Tóth P. HIV-1 Tat protein perturbs diacyglycerol production at the plasma membrane of neurosecretory cells during exocytosis. Commun Integr Biol 2013 ; 6 (5) : e25145.

10. Debaisieux S, Rayne F, Yezid H, Beaumelle B. The ins and outs of HIV-1 Tat. Traffic $2012 ; 13: 355-63$. 